\title{
PHOTOMETRIC CLASSIFICATION OF BLUE HORIZONTAL-BRANCH STARS
}

\author{
A. G. DAVIS PHILIP* \\ Dudley Observatory and State University of New York at Albany
}

\begin{abstract}
The Strömgren four-color system has been used to classify blue horizontal-branch stars in globular clusters and in the general field. A relation between $\delta \log g\left(\log g_{\text {main sequence }}-\log g_{\text {star }}\right)$ and $\delta c_{1}\left(c_{1}\right.$ observed $-c_{1}$ main sequence $)$ has been determined which gives $\log g$ to within \pm 0.2 for stars with $\log g$ 's between 4.4 and 2.0. Newell (1970) has determined a relation between $(B-V)_{0}$ and $\theta_{\mathrm{e}}$. Thus the gravities and effective temperatures of blue horizontal-branch stars can be determined from photometric measures alone. Five globular clusters have been studied at the present time. The BHB stars in the globular cluster M 3 and M 13 have lower surface gravities than BHB stars in the other clusters studied. Iben and Rood (1970) have recently suggested that the BHB stars of M3 may have lost more mass than BHB stars in more metal poor clusters.
\end{abstract}

\section{Introduction}

The purpose of this preliminary report is twofold. First, it will be shown how blue horizontal-branch (BHB) stars can be classified by photometric means and, second, the relationship of the field horizontal-branch (FHB) stars to the BHB stars will be indicated.

In the course of a study of galactic structure at high galactic latitudes, finding lists of early-type stars in a number of selected regions (this volume, p. 295) have been compiled. Among the fainter stars in each list, a natural group of stars has been found with the common characteristics of large Balmer jumps and low metal abundances. Since these qualities are similar to blue stars found in globular clusters, a program of measuring a number of BHB stars in a number of globular clusters with a large range in metal abundance has been started. Sufficient observations have been made of BHB and FHB stars that certain conclusions can now be drawn.

\section{Field Horizontal-Branch Stars}

Before discussing the four-color photometry of BHB stars, it would be useful to point out some relations derived among temperature, color, luminosity, and other parameters. In the range of temperature, $0.56<\theta_{e}<0.72$ it has been shown (Newell, 1970) that horizontal-branch stars have a different relation in the $\theta_{e},(B-V)_{0}$, plane than do Population I stars, in the sense that for the same $(B-V)_{0}$, the BHB stars have lower effective temperatures. The relation for BHB stars is shown in Figure 1 (Figure 3 from Newell, 1970) and is the one used in this study. Mihalas (1970) has computed the strength of the Balmer jump from LTE model atmospheres as a function of effective

* Visiting Astronomer, Kitt Peak National Observatory and Cerro Tololo Inter-American Observatory, which are operated by the Association of Universities for Research in Astronomy, Inc., under contract with the National Science Foundation. 


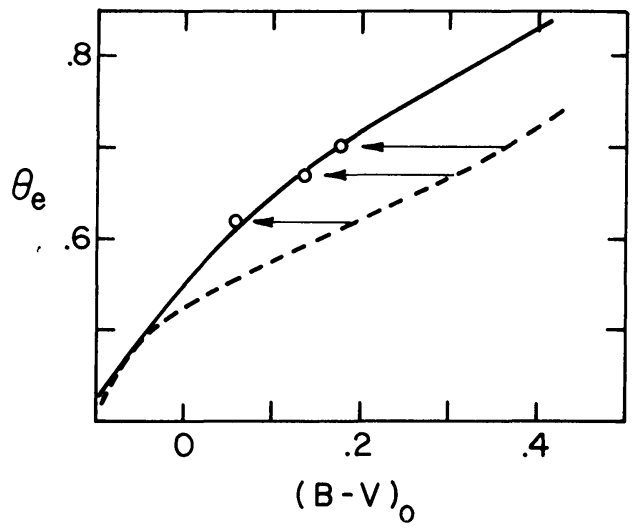

Fig. 1. $\theta_{e}$ vs $(B-V)_{0}$ (Figure 3 from Newell, 1970). The solid line represents the $\theta_{e},(B-V)_{0}$ calibration for BHB stars from Newell et al. (1969). The dashed line represents the Morton and Adams (1968) main sequence.

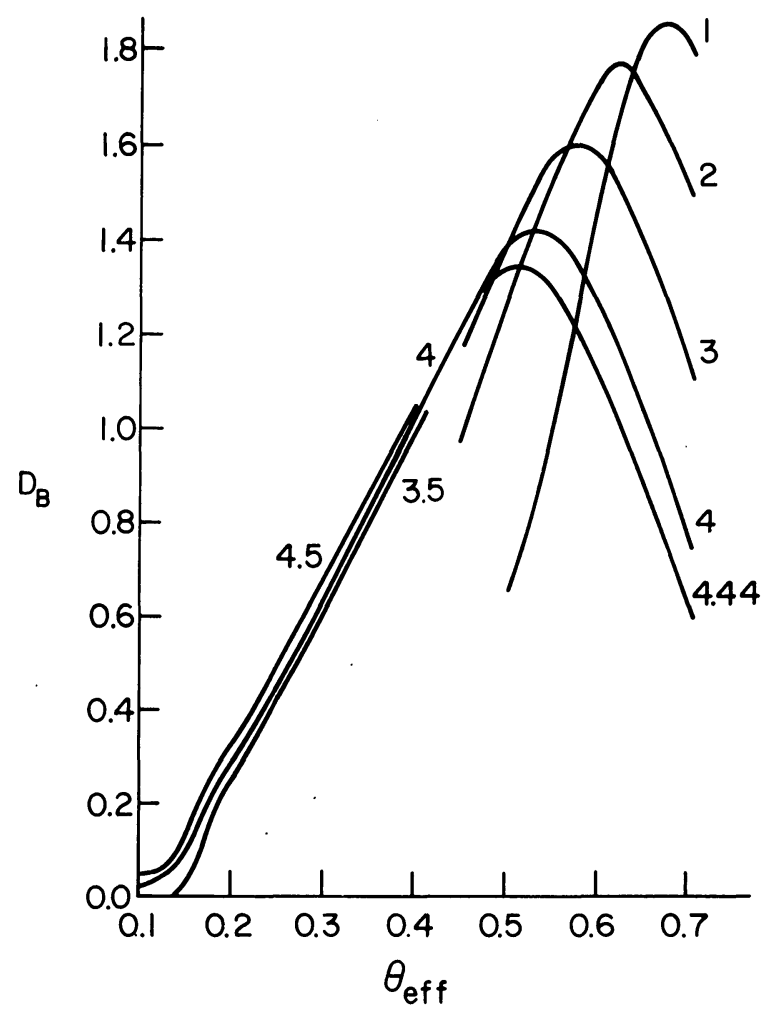

Fig. 2. Balmer groups computed from LTE model atmospheres, as a function of effective temperature and gravity. (Figure 6-3 from Mihalas, 1970). The Balmer jump, in magnitudes, is plotted vs the effective temperature. The curves are labeled with $\log g$. 
temperature and gravity. For $0.56<\theta_{e}<0.72$ there appears to be a linear relationship between changes in $\log g$ (between $\log g=4.4$ and 3.0) and $D_{B}$, the magnitude of the Balmer jump. This relationship is shown in Figure 2. (Figure 6-3 from Mihalas, 1970). The $c_{1}$ index $\left[c_{1}=(u-v)-(v-b)\right]$ is a measure of the Balmer jump and thus should be a good measure of $\log g$ in this temperature range, if the temperature is first determined by some other method. The $m_{1}$ index $\left[m_{1}=(v-b)-(b-y)\right]$ has been shown to be a measure of the metal abundance in the range of effective temperatures being considered. The $u$ and $v$ filters are affected by blanketing while the $b$ and $y$ filters are relatively unaffected. Thus a metal-rich star of the same effective temperature as a metal-poor star will have a different $m_{1}$ index.

Since $\theta_{e}, \log g$, and a measure of the metal abundance can be obtained from a study of the four-color indices, the Strömgren four-color system is an excellent system for the study of BHB stars.

The first stars to be measured were four prototype FHB stars, HD 2857, 86986, 109995, and 161817, listed by Oke et al. (1966). Matching photoelectric spectrum scans to atmospheric models they obtained estimates of $\log g$ and $\theta_{\boldsymbol{e}}\left(\log g=3.0, \theta_{\boldsymbol{e}}=0.6\right)$ which indicated that these stars were FHB stars. Three of these stars $(86986,109995,161817)$ have been analyzed for chemical abundance (Kodaira, 1964; Wallerstein and Hunziker, 1964; and Kodaira, 1969) and were found to have metal abundances, $\mathrm{Fe} / \mathrm{H}$, which were down by factors greater than 10 from $\mathrm{Fe} / \mathrm{H}$ values for Population I stars. Kodaira et al. (1969) also showed that these stars have large negative $V$ velocities and high $W$ velocities. These stars have been set up as secondary standards in the four-color and $\mathrm{H} \beta$ systems. The standards values are shown in Table I along with the ZAMS values for an A0 star for comparison.

For early A stars, it can be seen that the $c_{1}$ index of a FHB star is about 0.2 higher, the $m_{1}$ index about 0.04 lower, and the $\mathrm{H} \beta$ index about 0.1 lower than a main sequence star. Some of these differences can be seen by inspection of the spectra shown in Figure 3 (Figure 5 of Philip, 1968). Spectra, at a dispersion of $128 \AA \mathrm{mm}^{-1}$ of two of the prototype FHB stars are shown at the top of the figure. In the middle are spectra of two Population I stars and on the bottom are spectra of two FHB stars found at high galactic latitudes. The Balmer lines of the FHB stars are sharper than the Balmer lines in the Population I spectra and more Balmer lines can be seen in the FHB spectra. On spectra taken in the $U V$ a stronger Balmer jump can be seen.

TABLE I

Four-color and $\mathrm{H} \beta$ indices for prototype field horizontal-branch stars

\begin{tabular}{lllllll}
\hline Star & $b-y$ & $c_{1}$ & $m_{1}$ & $n$ & $\beta$ & $n$ \\
\hline Population I A0 star & 0.000 & 1.070 & 0.171 & & 2.943 & \\
HD 109995 & 0.048 & 1.286 & 0.134 & 48 & 2.823 & 46 \\
HD 86986 & 0.089 & 1.266 & 0.123 & 30 & 2.822 & 25 \\
HD 161817 & 0.129 & 1.207 & 0.112 & 39 & 2.670 & 31 \\
HD 2857 & 0.135 & 1.213 & 0.114 & 36 & 2.684 & 29
\end{tabular}


Fifty FHB stars have been identified in areas at high galactic latitudes (Philip, 1967, 1968, 1969a, b, and 1970a). In five of the regions under study, sufficient A stars have been measured so that the color excess as a function of distance modulus has been determined, and thus the intrinsic colors of FHB stars found in these regions are easily calculated. Forty-five stars, in five regions (NGP, SGP, 1 HLF 2, 1 HLF 3, and

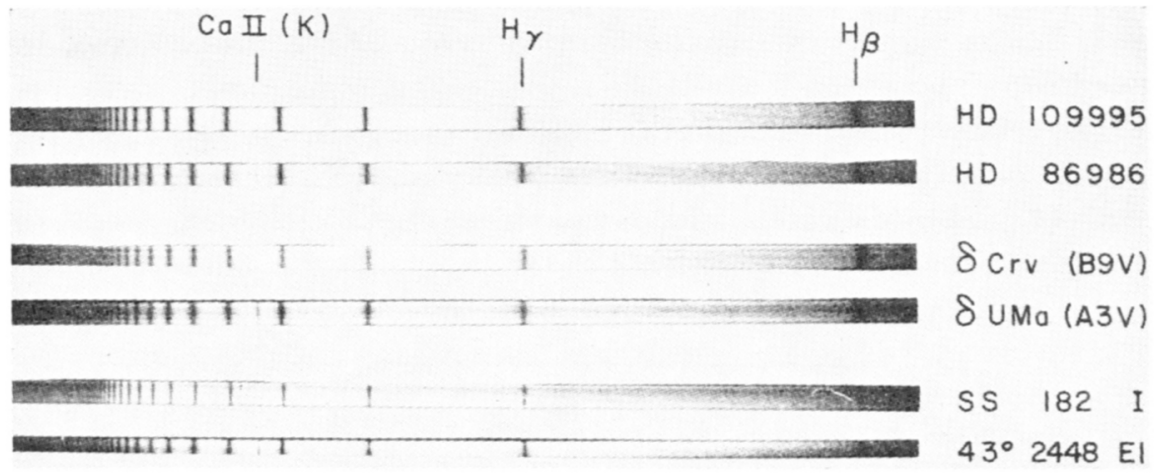

Fig. 3. Spectra of six stars. (Figure 5 from Philip, 1968). The upper two are of field horizontalbranch stars (Oke et al., 1966). The middle two are of two Population I main sequence stars.

The bottom two spectra are of two field horizontal-branch stars (Philip, 1968).

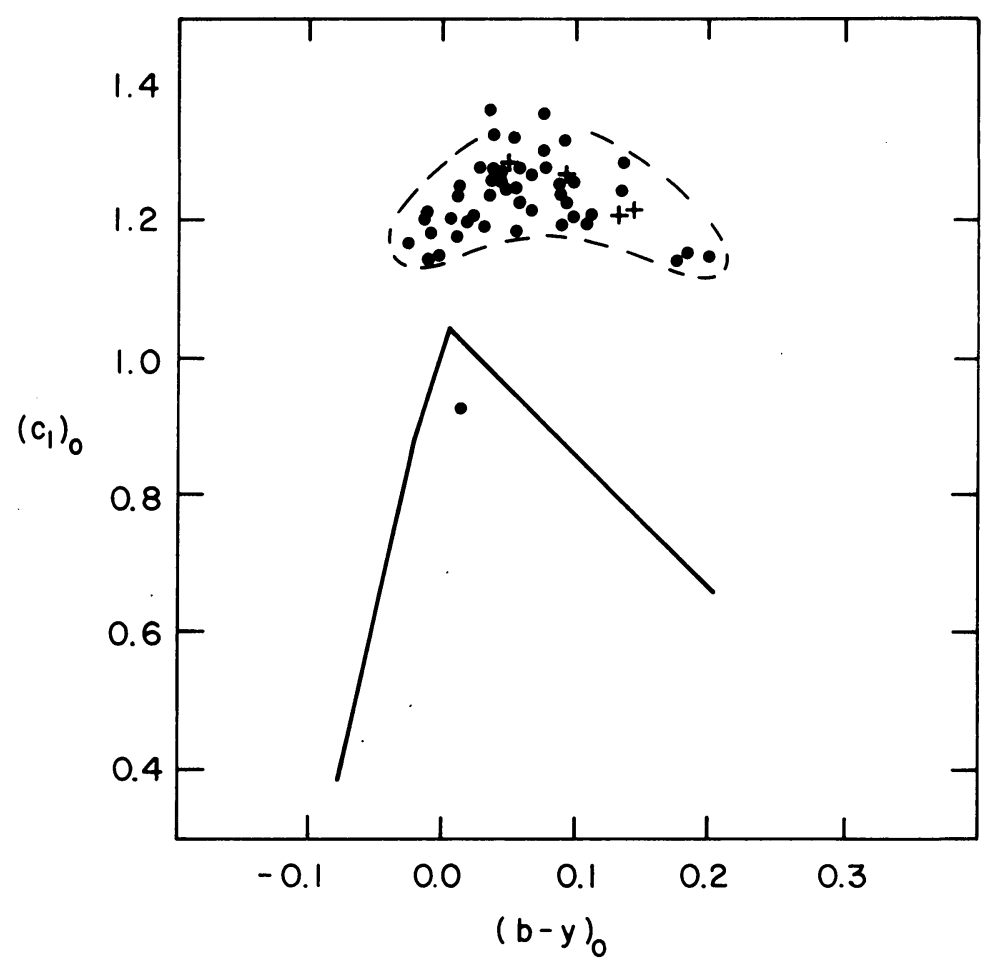

Fig. 4. The $\left(c_{1}\right)_{0}$ index vs $(b-v)_{0}$ for 45 FHB stars in regions at high galactic latitudes. The Solid line represents the zero age main sequence. The dotted line indicates the area in which the FHB stars fall. The crosses represent the indices of the four prototype FHB stars. 
4 HLF 6) have been measured in the four-color system and their intrinsic color indices are plotted in Figures 4 and 5 . The $\left(c_{1}\right)_{0}$ index is plotted against $(b-y)_{0}$, in Figure 4. The solid lines indicate the zero age main sequence for B stars (Slettebak et al., 1968) and A stars (Crawford, 1970). The indices for the four prototype FHB stars are plotted as crosses. The point falling below the ZAMS represents SS 182 I, a star identified by Slettebak and Stock (1959) as a FHB star. The rest of the stars fall in a common area. A dotted line has been drawn around these points to mark the area occupied by the natural group.

The $\left(m_{1}\right)_{0}$ index is plotted against $(b-y)_{0}$ in Figure 5. Again, the indices for the four prototype FHB stars are plotted as crosses. Dotted lines have been drawn around the area occupied by the natural group, an area nearly perpendicular to the zero age main sequence, indicated by the solid line. The two points that fall outside the area

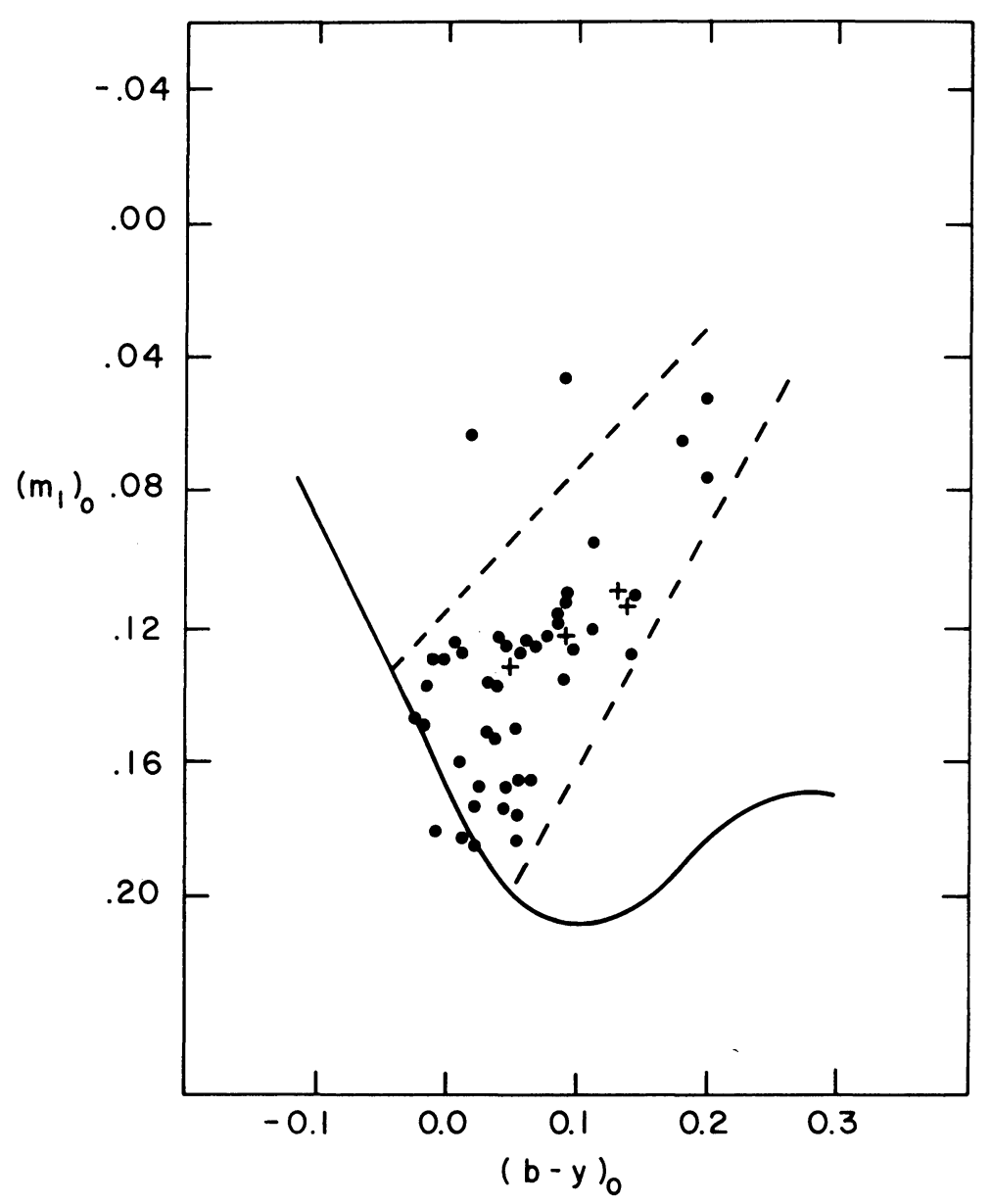

Fig. 5. The $\left(m_{1}\right)_{0}$ index vs $(b-y)_{0}$ for 45 FHB stars in regions at high galactic latitudes. The symbols are the same as in Figure 4. 
marked by the dotted lines are indices representing SS 182 I and another star with very high $c_{1}$ and very low $m_{1}$ indices.

The $\mathrm{H} \beta$ index is plotted against $(b-y)_{0}$ for the group of stars in Figure 6. Four crosses represent the prototype FHB stars. The $\mathrm{H} \beta$ indices of the FHB stars generally fall below the zero age main sequence. In Figures 4, 5, and 6, the indices representing the four prototype and the FHB stars found in high galactic latitude areas fall in the same areas. The position occupied by the points in Figure 4 is an effect of higher luminosity or lower surface gravity. The $u$ magnitude is depressed due to the larger Balmer jump and the $v$ magnitude is slightly greater due to the smaller blanketing, both of which lead to a higher $c_{1}$ index. In Figure 5 , the position occupied by the points representing FHB stars is caused by two effects. One is a luminosity effect due to the $\mathrm{H} \delta$ line which falls in the middle of the passband of the $v$ filter. The other is the effect of a lower metal abundance in FHB stars which decreases the blanketing thus causing a brighter $v$ magnitude and a smaller $m_{1}$ index. In Figure 6 , the position of the points representing the $\mathrm{H} \beta$ indices of $\mathrm{FHB}$ stars is in the same sense as the variations in the Balmer lines seen in Figure 3.

Plots of $\left(m_{1}\right)_{0}$ vs $(b-y)_{0}$ could be better indicators of metal abundance variations were it possible to reduce the scatter in a diagram such as Figure 2. The presence of $\mathrm{H} \delta$ in the $v$ passband introduces difficulties of interpretation which has led to the introduction of a new $v^{1}$ filter to be added to the Strömgren system. The $v^{1}$ filter has a central wavelength of $4220 \AA$ and a half width of $45 \AA$. A new $m_{1}$ index will be computed using the $v^{1}$ filter which should be a better measure of the metal abundance.

\section{Radial Velocities of Field Horizontal Branch Stars}

Radial velocities have been measured for 33 FHB stars, 18 by Philip (1969c, 1970b) and 15 by Rodgers (1971). The velocity measures are presented in Table II.

The velocity dispersion for the group in Table II, for which $V>10.0$, is $\pm 113 \mathrm{~km} \mathrm{~s}^{-1}$. Woolley and Stewart (1967) observed stars in the NGP region and found $\sigma= \pm 18 \mathrm{~km} \mathrm{~s}^{-1}$ for A stars with $8<\mathrm{V}<10$. For brighter A stars at the NGP, $\sigma= \pm 8 \mathrm{~km} \mathrm{~s}^{-1}$, thus there is a rapid increase in $\sigma$ with increasing $Z$.

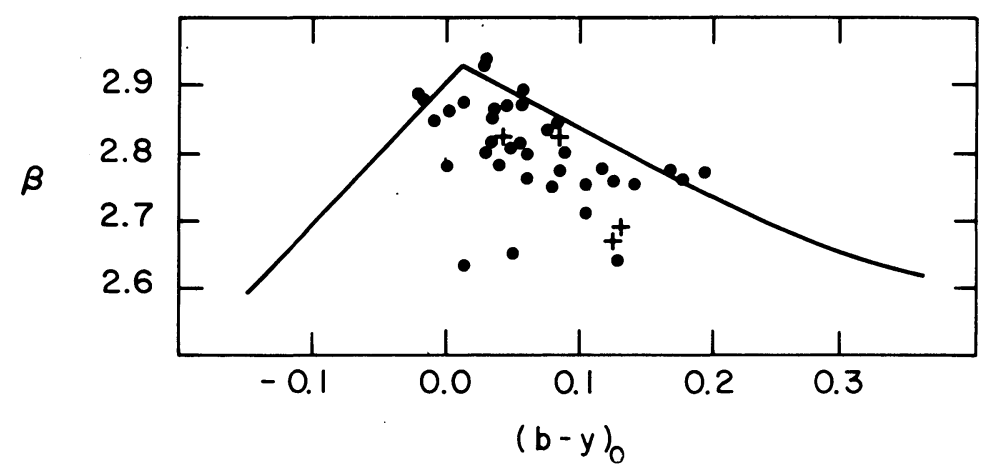

Fig. 6. The $\beta$ index vs $(b-y)_{0}$ for 36 FHB stars. The symbols are the same as in Figure 4. 
TABLE II

Radial velocities of field horizontal branch stars

\begin{tabular}{|c|c|c|c|c|c|}
\hline \multicolumn{2}{|l|}{ Star } & \multirow{2}{*}{$\begin{array}{l}\text { Area } \\
\text { NGP }\end{array}$} & \multirow{2}{*}{$\begin{array}{l}\begin{array}{l}V \\
\mathrm{~km} \mathrm{~s}^{-1}\end{array} \\
-143\end{array}$} & $\begin{array}{l}W(K) \\
\text { in } \AA\end{array}$ & \multirow{2}{*}{$\begin{array}{l}\text { Source } \\
1\end{array}$} \\
\hline SS 182 & I & & & & \\
\hline SS 287 & I & NGP & 123 & & 1 \\
\hline SS 194 & II & NGP & 36 & & 1 \\
\hline SS 197 & II & NGP & 150 & & 1 \\
\hline SS 202 & II & NGP & 123 & & 1 \\
\hline SS 227 & II & NGP & 83 & & 1 \\
\hline SS 229 & II & NGP & -90 & & 1 \\
\hline $50^{\circ} 2236$ & $6 \mathrm{El}$ & 1 HLF 3 & -89 & & 1 \\
\hline $47^{\circ} 2324$ & $+\mathrm{Nl}$ & 1 HLF 3 & -56 & & 1 \\
\hline $43^{\circ} 2448$ & $\mathrm{El}$ & 1 HLF 5 & -230 & & 1 \\
\hline $40^{\circ} 2179$ & $\mathrm{El}$ & 4 HLF 3 & 70 & & 1 \\
\hline $40^{\circ} 2325$ & $5 \mathrm{Nl}$ & 4 HLF 5 & 83 & & 1 \\
\hline S 10 & & 1 HLF 2 & -160 & & 2 \\
\hline $13 \quad 110$ & & 1 HLF 2 & -123 & & 2 \\
\hline 16111 & & 1 HLF 2 & -253 & & 2 \\
\hline $17 \quad 24$ & & 1 HLF 2 & 76 & & 2 \\
\hline $17 \quad 136$ & & 1 HLF 2 & -212 & & 2 \\
\hline 1821 & & 1 HLF 2 & -53 & & 2 \\
\hline PS 1 & II & SGP & -64 & $<0.4$ & 3 \\
\hline PS 5 & II & SGP & -132 & $<0.4$ & 3 \\
\hline PS 6 & II & SGP & -51 & $<0.4$ & 3 \\
\hline PS 16 & II & SGP & -215 & 0.7 & 3 \\
\hline PS 23 & II & SGP & 92 & 1.2 & 3 \\
\hline PS 25 & II & SGP & -32 & 0.7 & 3 \\
\hline PS 27 & II & SGP & -100 & 2.9 & 3 \\
\hline PS 35 & II & SGP & -51 & 0.6 & 3 \\
\hline PS 36 & II & SGP & 106 & 1.7 & 3 \\
\hline PS 45 & II & SGP & -2 & 0.4 & 3 \\
\hline PS 50 & II & SGP & 147 & 0.5 & 3 \\
\hline PS 52 & II & SGP & -34 & $<0.4$ & 3 \\
\hline PS 53 & II & SGP & 82 & $<0.4$ & 3 \\
\hline PS 57 & II & SGP & 6 & 3.3 & 3 \\
\hline PS 59 & II & SGP & 26 & 0.4 & 3 \\
\hline
\end{tabular}

a Sources: 1, Philip (1969c); 2, Philip (1970b); 3, Rodgers (1971).

\section{Equivalent Width of the Calcium $K$ Line}

Rodgers (1971) published spectrographic measures of the faint A stars at the SGP from the list of Philip and Sanduleak (1968). One of the measures was the equivalent width of the calcium $\mathrm{K}$ line. $W(\mathrm{~K})$ is plotted against $(B-V)$ for the faint $\mathrm{A}$ stars measured by Rodgers (1971, his Figure 2) in the top part of Figure 7. The main point of his graph was that there are a number of faint $A$ stars far below the galactic plane with normal calcium abundance. In the lower graph the figure is replotted this time showing only the stars from Table II, which have all been classified as FHB stars on the basis of four-color and $\mathrm{H} \beta$ photometry. The great majority of these stars have much smaller 
equivalent widths than is normal for a Population I star. This evidence, along with the high velocity dispersion, tends to confirm that FHB stars are members of Population II.

\section{Stellar Densities at the Galactic Poles}

Since the samples of early-type stars at the galactic poles have been selected in an unbiased way and are complete to a limiting visual magnitude fainter than 13 th mag., the stellar density of these stars can be compiled, knowing the volume of the area searched. The number of stars per $10^{6} \mathrm{pc}^{3}$ is plotted against the perpendicular distance from the galactic plane in kiloparsecs in Figure 8. The densities of A0 and A2-A7 stars from Upgren (1963) are plotted as solid lines in the upper part of the diagram. The line across the bottom of the diagram represents the density of RR Lyrae stars at the NGP found by Kinman et al. (1966). The densities of the FHB stars are plotted as circles (NGP) and crosses (SGP). For distances less than two kiloparsecs accurate densities can not be calculated because the volume of the cone being investigated is small and the density low. For distances greater than five kiloparsecs the magnitudes
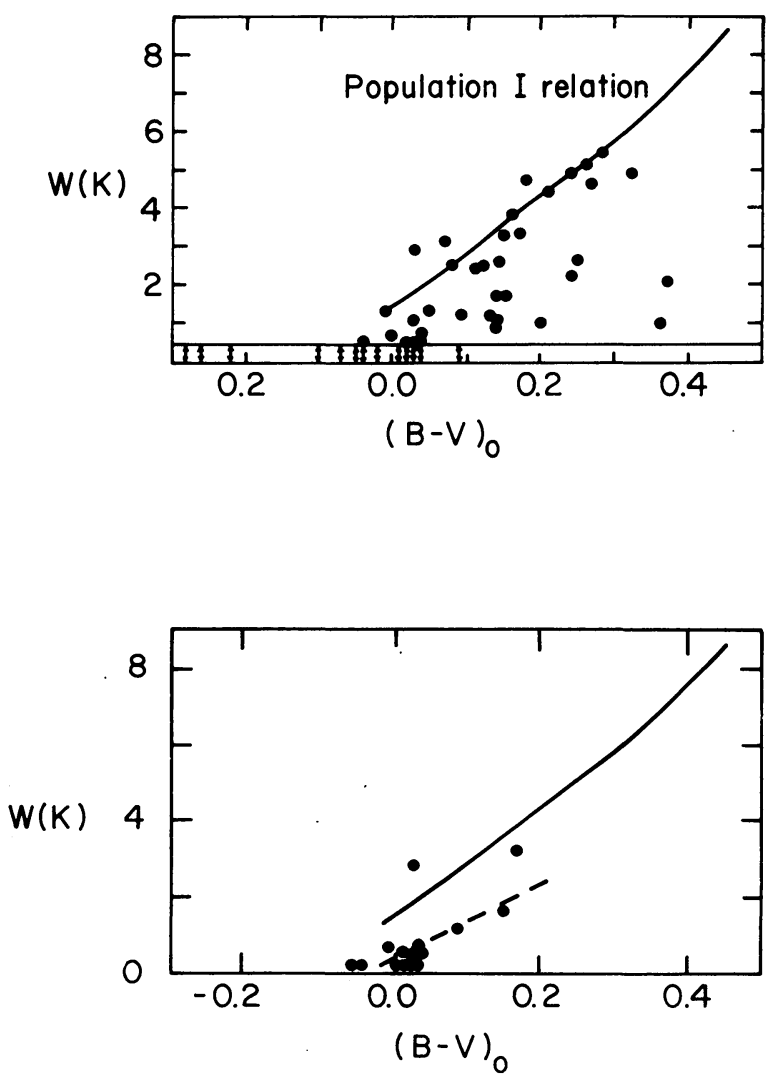

Fig. 7. $W(\mathrm{~K})$, the equivalent width of the calcium $\mathrm{K}$ line, vs $(B-V)_{0}$. The upper figure is Figure 2 from Rodgers (1971). The lower figure presents the indices for FHB stars at the SGP. 
of the stars would be below the plate limit and thus the data are incomplete. In between these two limits, where a complete count is being made of the blue halo stars, the space density of the FHB stars is approximately 10 times that of the R R Lyrae stars, a situation similar to that found in many globular clusters.

\section{Blue Horizontal Branch Stars in Globular Clusters}

Observations have been made of BHB stars in the globular clusters M3, M4, M13,

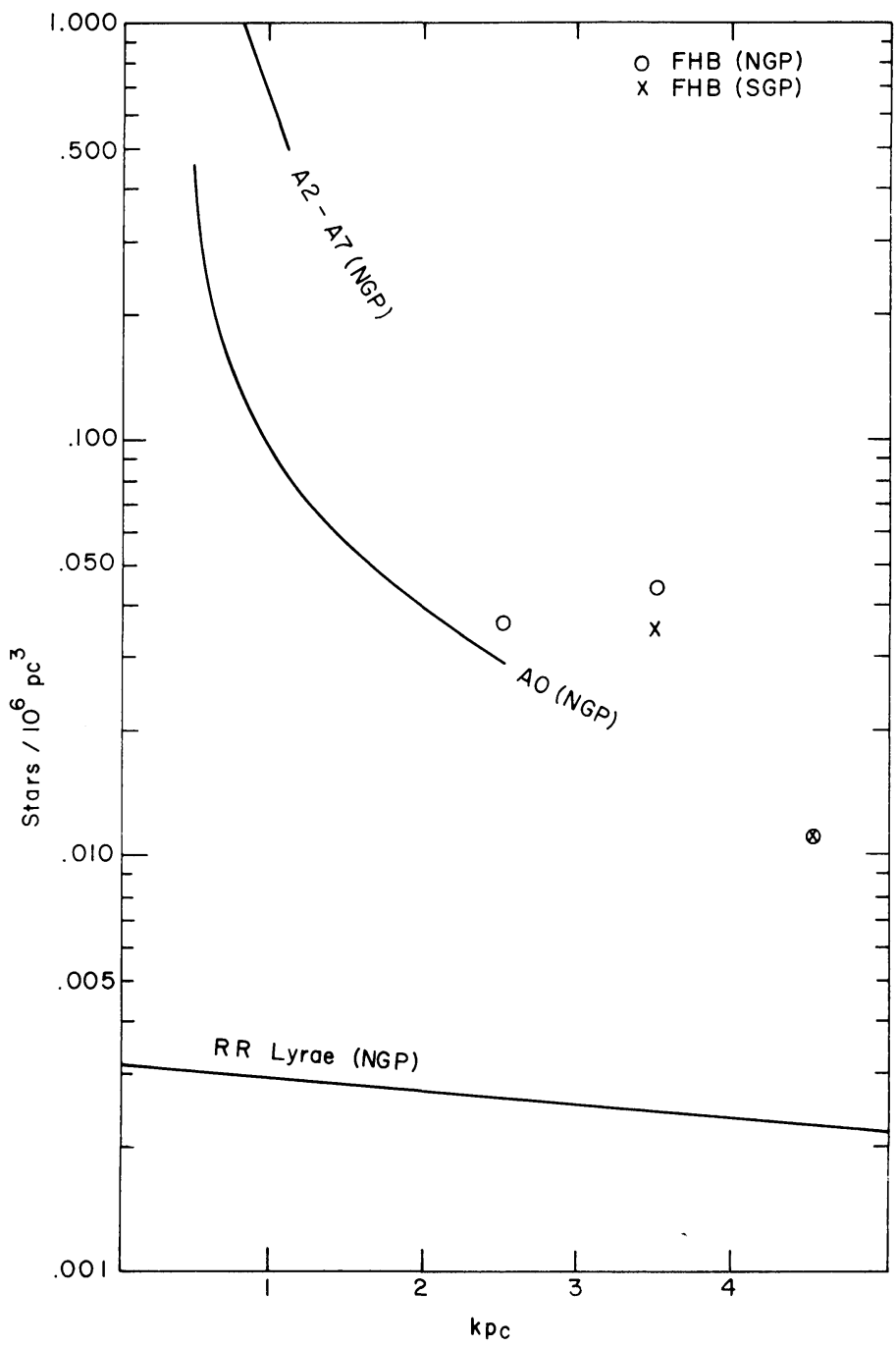

Fig. 8. The stellar density (stars per $10^{6} \mathrm{pc}^{3}$ ) as a function of the distance from the galactic plane in kiloparsecs. At 3-4 kpc, the FHB stars have densities approximately ten times that of the RR Lyrae stars. 
M55, M92, and NGC 6397. These stars are quite faint, reaching a $V$ magnitude of 16.2 , and thus the precision with which the four-color indices can be measured is less than that of the FHB stars, which are not fainter than $V=14$. In spite of this handicap, certain similarities can be seen between the FHB and BHB stars, as is shown in the following graphs. The Balmer jump index, $\left(c_{1}\right)_{0}$ is plotted against $(b-y)_{0}$ in Figure 9. The solid line indicates the zero age main sequence, the dotted line indicates the area occupied by the FHB stars. Stars in two of the clusters studied, M3 and M13, of intermediate metal abundance, are represented by circles; stars in other clusters are represented by dots. The distribution of globular cluster BHB stars is quite similar to that for the FHB stars.

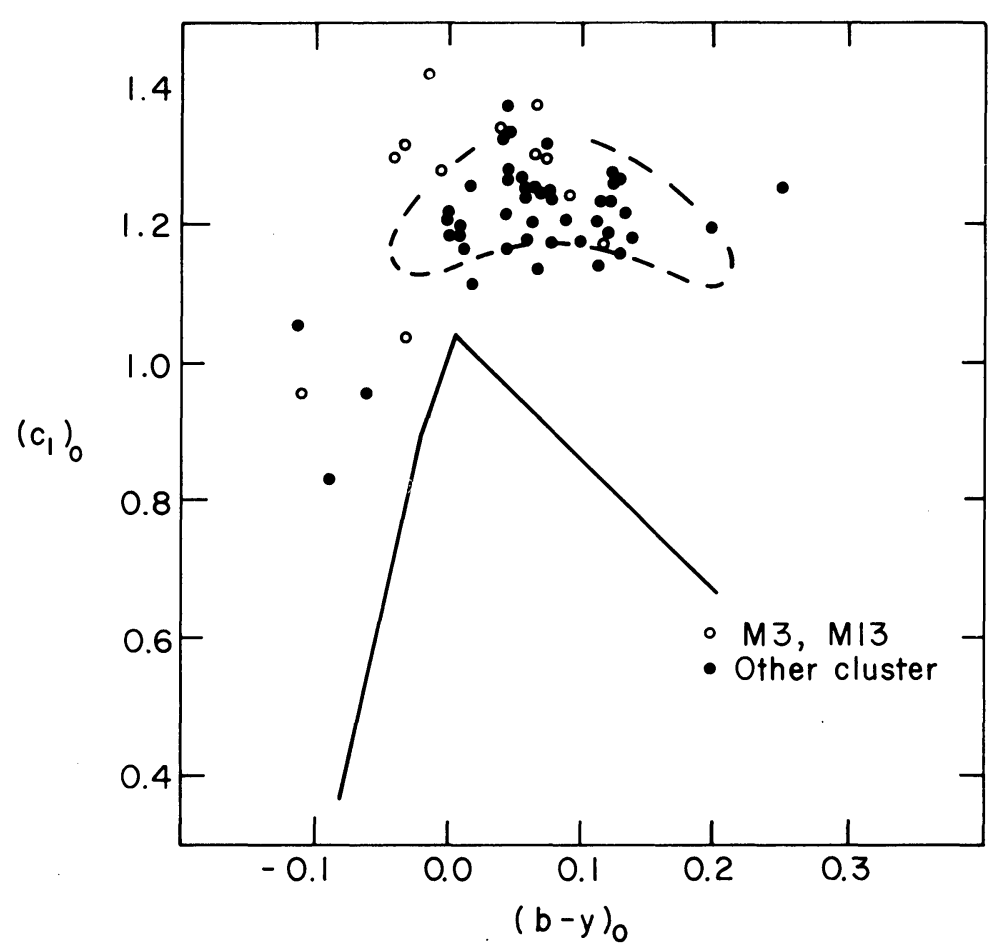

Fig. 9. The $\left(c_{1}\right)_{0}$ index vs $(b-y)_{0}$ for BHB stars in globular clusters. The $\left(c_{1}\right)_{0}$ indices for BHB stars in M3 and M13 are larger, near $(b-y)_{0}=0.00$, than $\left(c_{1}\right)_{0}$ indices for other clusters.

There are some bluer BHB stars which fall outside the area marked by the dotted lines; no stars this blue were found in the field (except possibly SS $182 \mathrm{I})$. At $(b-y)_{0}=$ $=0.00$ there are some points representing stars in M3 and M13 which fall higher than the dotted line. This effect can be seen more clearly in Figure 10, where mean lines have been drawn through all the points representing the two types of clusters. The higher $\left(c_{1}\right)_{0}$ index of the intermediate metal abundance clusters seems quite real.

The metal index, $\left(m_{1}\right)_{0}$ is plotted against $(b-y)_{0}$ in Figure 11. The solid and dotted 
lines have the same meaning as in Figure 9. There does not seem to be any difference between the two types of clusters in this diagram; all the cluster points fall in the same area as the FHB stars. As noted earlier, the $m_{1}$ index is complicated by the presence of $\mathrm{H} \delta$ in the $v$ passband. The index does not discriminate between stars of low metal abundance but works best between a solar metal abundance and one tenth solar abundance.

\section{The $\log g, \theta_{e}$ Relation}

A search of the literature has yielded 34 early-type stars* for which $\log g$ and $\theta_{e}$ have been determined by means of high dispersion spectra or photoelectric spectrum scans

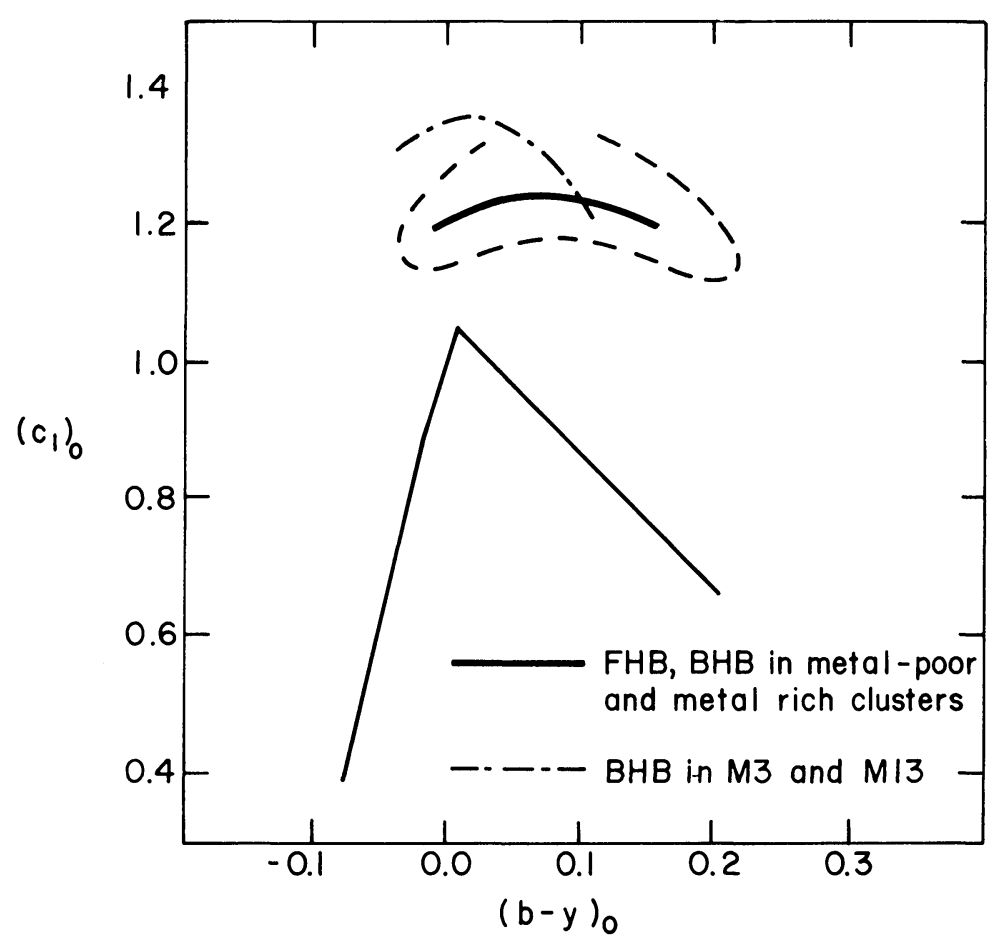

Fig. 10. The mean $\left(c_{1}\right)_{0}$ index as a function of $(b-y)_{0}$ for intermediate metal abundance clusters and other clusters.

matched to atmospheric models. Four-color data exists for these stars; either in the Strömgren-Perry Catalogue (1962) or in the author's unpublished catalogue of FHB stars. In Figure 12, (Figure 1 of Philip, 1972) $\delta \log g$ is plotted against $\delta c_{1}$ for each of the 34 stars. The dotted line represents a theoretical relation derived by Strom (1970) between $\delta \log g$ and $\delta c_{1}$. The observational points fall about this relation and indicate that theory and observation agree quite well. The $\delta \log g$ can be estimated from the

* A list of these stars will be published elsewhere (Philip, 1972). 
$\delta c_{1}$ with a probable error of \pm 0.2 in $\log g$. Using this relation, one can compute $\log g$ for the BHB stars in the globular clusters so far investigated.

In Figure 13 (Figure 2 of Philip, 1972) the solid line represents the mean relation between $\log g$ and $\theta_{e}$ found for NGC 6397 (Newell et al., 1969), while the symbols represent the results obtained here. The small symbols represent BHB stars in M4, M55, and M92 ( a metal-rich and two metal-poor clusters). These points scatter about

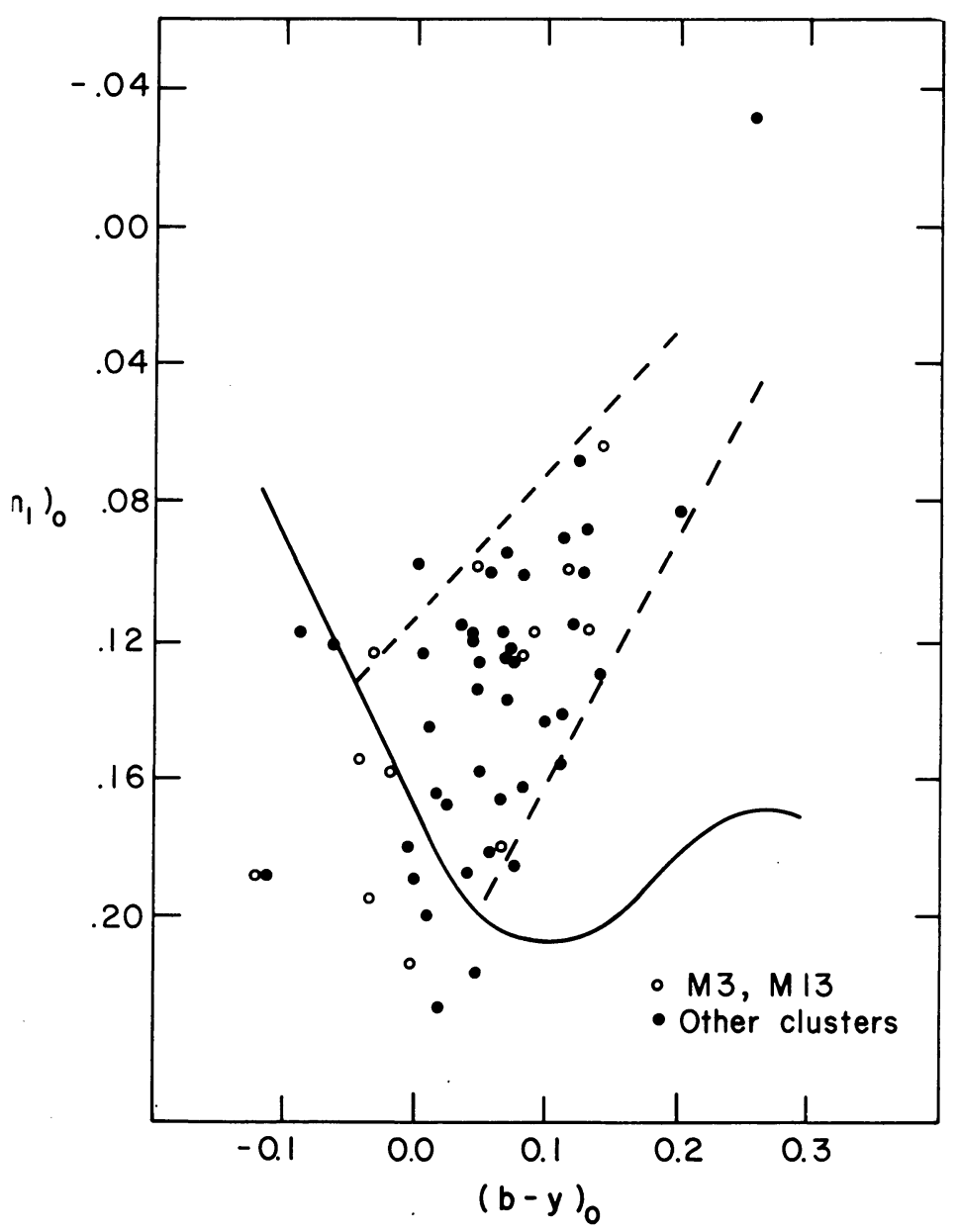

Fig. 11. The $\left(m_{1}\right)_{0}$ index vs $(b-y)_{0}$ for BHB stars in globular clusters.

the mean relation for NGC 6397. Large symbols, representing BHB stars in M3 and M13, fall above the mean relation (in the same sense as the higher $\left(c_{1}\right)_{0}$ indices in Figure 10) and scatter about a (dotted) line which is about 0.3 higher than the mean relation. This behavior would be expected if the BHB stars in intermediate metal abundance clusters have lost $0.2 M_{\odot}$ and the radii have changed by $20 \%$. 


\section{Conclusion}

The FHB stars have been shown to be metal-poor, to have a high velocity dispersion, and to have similar colors to BHB stars in globular clusters. The hypothesis that the FHB stars are indeed BHB stars in the general field seems to be confirmed in light of all these data. More observations of additional stars and more observations per star are needed to confirm the low surface gravities of BHB stars in globular clusters of inter-

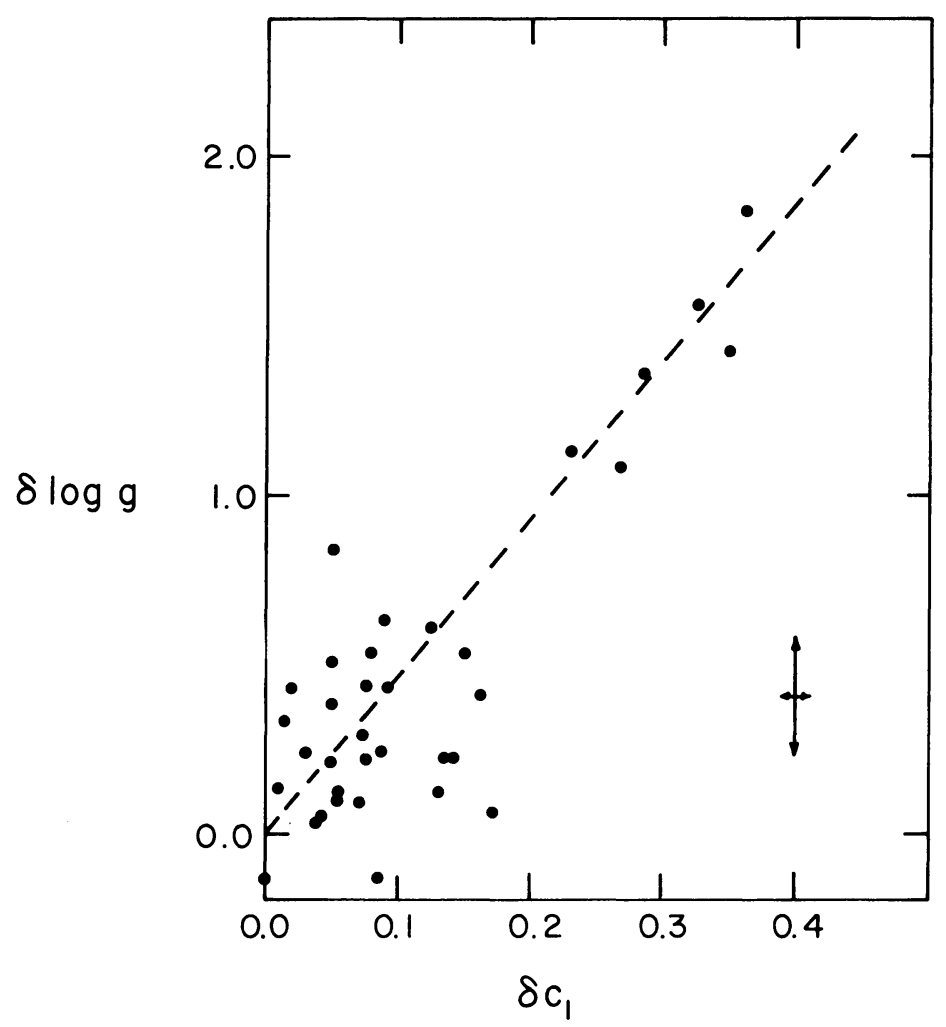

Fig. 12. $\log g$ vs $\delta c_{1}$ for 34 early type stars for which $\theta_{\mathrm{e}}$ and $\log g$ have been obtained by means of high dispersion spectra or photoelectric scans.

mediate metal abundance. If these additional data agree with the preliminary data, they will be an interesting confirmation of the hypothesis of Iben and Rood (1970) that BHB stars in clusters of intermediate metal abundance have lost more mass than BHB stars in metal-poor clusters.

\section{Acknowledgements}

The Directors of the Cerro Tololo Inter-American Observatory, Kitt Peak National Observatory, and Steward Observatory are thanked for making time available on the 


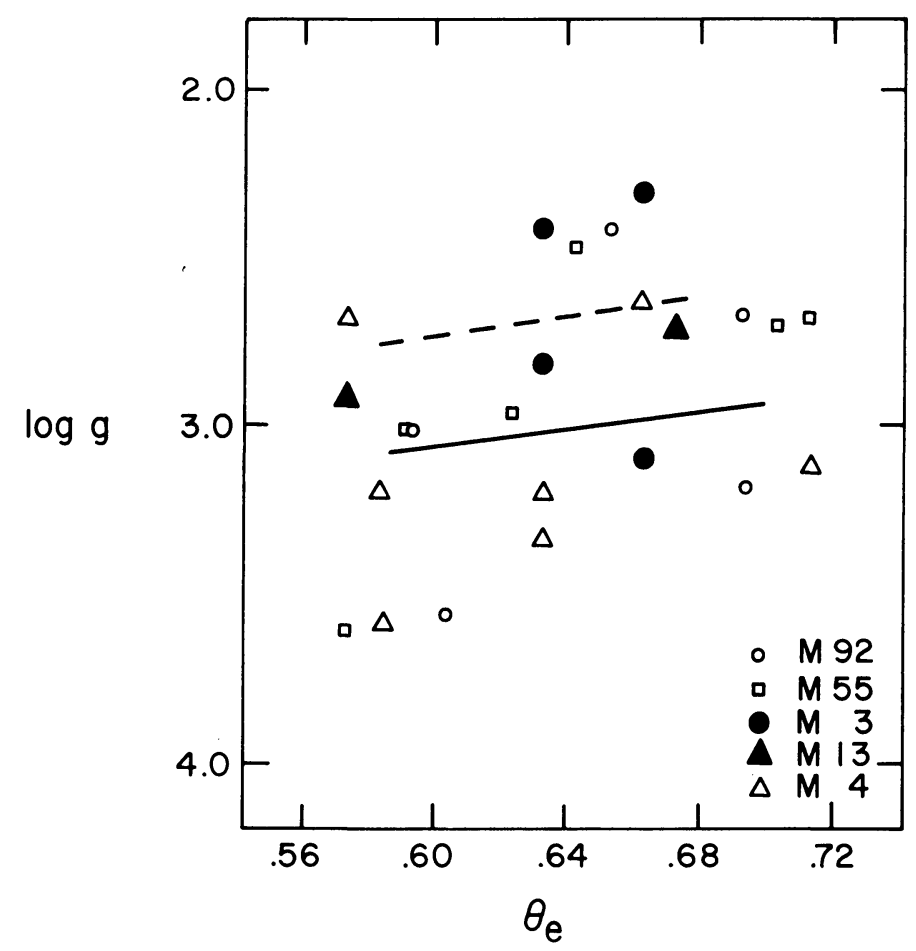

Fig. 13. $\log g$ vs $\theta_{\mathrm{e}}$ for BHB stars in five globular clusters. The line represents the mean relation for NGC 6397 (Newell, Rodgers, and Searle, 1969). The intermediate metal abundance clusters M3 and M13 have BHB stars with $\log g$ 's about 0.3 smaller than the mean relation.

60-in., 36-in., 84-in., and 90-in. telescopes for this project. The National Science Foundation and the SUNY Research Foundation have partially supported this project.

\section{References}

Crawford, D. L.: 1970, in A. Slettebak (ed.), Stellar Rotation, D. Reidel Publ. Co., Dordrecht, Holland, p. 204.

Iben, I and Rood, R. T.: 1970, Astrophys. J. 161, 587.

Kinman, T. D., Wirtanen, C. A., and Janes, K. A.: 1966, Astrophys. J. Suppl. 13, 379.

Kodaira, K.: 1964, Z. Astrophys. 59, 139

Kodaira, K., Greenstein, J. L., and Oke, J. B.: 1969, Astrophys. J. 155, 525.

Mihalas, D.: 1970, Stellar Atmospheres, W. H. Freeman and Co., San Fransisco, p. 190.

Newell, E. B.: 1970, Astrophys. J. 159, 443.

Newell, E. B., Rodgers, A. W., and Searle, L.: 1969, Astrophys. J. 156, 597.

Oke, J. B., Greenstein, J. L., and Gunn, J.: 1966, in R. F. Stein and A. G. W. Cameron (eds.), Stellar Evolution, Plenum Press, New York.

Philip, A. G. D.: 1967, Astrophys. J. 148, L143.

Philip, A. G. D.: 1968, Astrophys. J. 152, 1107.

Philip, A. G. D.: 1969a, Astron. J. 74, 209.

Philip, A. G. D.: 1969b, Astron. J. 74, 812.

Philip, A. G. D.: 1969c, Astrophys. J. Letters 158, 113.

Philip, A. G. D.: 1970a, Astron. J. 75, 957. 
Philip, A. G. D.: 1970b, Astron. J. 75, 246.

Philip, A. G. D.: 1972, Astrophys. J. 171, L51.

Philip, A. G. D. and Sanduleak, N.: 1968, Bol. Obs. Tonantzintla y Tacubaya 4, 253.

Rodgers, A. W.: 1971, Astrophys. J. 165, 581.

Slettebak, A. and Stock, J.: 1959, Astron. Abh. Hamburg, No. 5.

Slettebak, A., Wright, R. R., and Graham, J. A.: 1968, Astron. J. 73, 152.

Strom, S.: 1970, private communication.

Strömgren, B. and Perry, C. L.: 1962, unpublished.

Upgren, A. R.: 1963, Astron. J. 68, 475.

Wallerstein, G. and Hunziker, W.: 1964, Astrophys. J. 140, 214.

Woolley, R. and Stewart, J. O.: 1967, Monthly Notices Roy. Astron. Soc. 136, 329. 